\title{
Protée
}

\section{La voix chantée du silence}

\section{Simon Harel}

Volume 28, numéro 2, 2000

\section{Le silence}

URI : https://id.erudit.org/iderudit/030590ar

DOI : https://doi.org/10.7202/030590ar

Aller au sommaire du numéro

\section{Éditeur(s)}

Département des arts et lettres - Université du Québec à Chicoutimi

\section{ISSN}

0300-3523 (imprimé)

1708-2307 (numérique)

Découvrir la revue

\section{Citer cet article}

Harel, S. (2000). La voix chantée du silence. Protée, 28(2), 17-24.

https://doi.org/10.7202/030590ar

\section{Résumé de l'article}

De nombreux travaux ont été consacrés à la mise en jeu de l'autobiographie dans l'oeuvre de Michel Leiris. La très grande majorité de ces recherches ont mis l'accent sur l'association du code scripturaire et du référent corporel, ce dernier tenant lieu de matrice contenante de l'élan créateur. Cet article propose une réflexion sur la sémiotisation de l'oralité et du silence dans les écrits tardifs que sont Opérratiques et À cor et à cri. De façon très significative, ces écrits de Michel Leiris renouent avec une préoccupation poétique fondamentale abandonnée en 1925. Que signifie ce passage du regard à la voix du silence dans l'oeuvre tardive de Michel Leiris ? Comment comprendre l'abandon progressif des représentations du monde théâtral et tauromachique, associées chez Leiris à l'espace autobiographique, au profit du temps de la voix qui devient la forme vive et silencieuse du récit de soi ?
Ce document est protégé par la loi sur le droit d'auteur. L'utilisation des services d'Érudit (y compris la reproduction) est assujettie à sa politique d'utilisation que vous pouvez consulter en ligne.

https://apropos.erudit.org/fr/usagers/politique-dutilisation/ 


\section{LA VOIX CHANTÉE DU SILENCE}
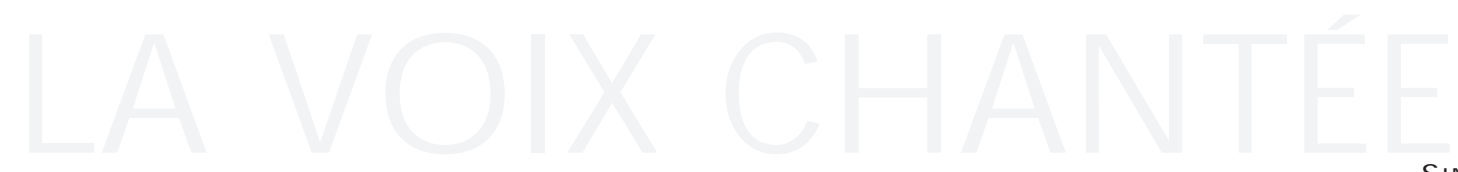

SIMON HAREL

Vouloir déterminer de quelle manière, l'inerte s'animant et les mots prenant apparemment leur libre essor, on passe, lorsqu'on écrit, du parler au chanter, quand ce n'est de la nullité du silence à quelque chose qui d'emblée se révèle sensible comme un chant, est - je gage - aussi follement naïf que le désir que, complices, nous eûmes jadis l'un de mes deux frères et moi de saisir l'instant précis où, couchés le soir dans nos lits, nous passions de la veille au sommeil, chute dans le noir dont au réveil on sait qu'elle a eu lieu mais sans pouvoir connaître, tant la conscience était embuée, la façon dont le pas a été franchi. ${ }^{1}$

Que nous apprend cette citation de Leiris à partir de laquelle je désire introduire une réflexion sur l'ambivalence du témoignage autobiographique à propos du silence? La formulation peut sembler polémique: il n'y a pas de sémiotique du silence si l'on entend ainsi la nécessité de saisir un objet dont l'immatérialité est la caractéristique première. La saisie du silence, si l'on respecte une perspective sémiotique, est un contresens. Il n'existe pas un silence "pur», une origine silencieuse qui serait antérieure à l'émergence du langage. C'est plutôt la réversibilité du signe ${ }^{2}$ qui nous permet d'aborder la question du silence. Le signe peut être perçu, dans un premier temps, comme la cristallisation d'une intention qui opacifie, par sa détermination obstinée à faire «sens», ce qui en constitue le pourtour. En somme, le signe, parce qu'il obéit à une logique bifocale ou encore réversible, énonce une intentionnalité qui inscrit la subjectivité dans la langue tout en tenant à distance le silence. Le signe est à sa façon une sépulture. Antonin Artaud ne s'était pas trompé sur cette mise en relation du signe et de la comptabilité funèbre qui tient lieu d'organisation sémiotique.

Tout système sémiotique se doit pour cette raison de créer du sens afin de faire échec à l'insensé qu'est le silence. Il n'est pas justifié d'étudier le silence sous la forme d'une «donnée» linguistique, surtout si l'on fait appel à la sémiotisation singulière du silence qu'impose la littérature, notamment dans sa dimension autobiographique. Tout discours, quels qu'en soient la singularité et l'auditoire potentiel, construit les formes signifiantes du silence et de la voix. Ainsi, l'oralité 
première, qui se donne à entendre comme manifestation non médiatisée de la voix, offre l'illusion d'une organisation signifiante qui est nourrie par le silence. C'est l'exemple de la tradition orale dont la structure énonciative est façonnée par cette mise en relief du silence qui a valeur de scansion. Le conteur qui habite un espace narratif qualifié de traditionnel sait que son discours possède une efficacité rhétorique dans la mesure où il est jalonné de séquences de silence. Cette efficacité rhétorique appartient au champ élargi de la sémiotique puisque le silence est personnalisé par un énonciateur qui peut en faire résonner les effets. Nous sommes alors au cœur d'un monde où prévaut le dialogue puisqu'il est entendu que le conteur s'adresse de manière délibérée à un auditoire dont il espère recevoir la reconnaissance de sa parole.

Il en va de même de la conversation dans la mesure où elle inscrit une pluralité de prises de parole. La conversation n'échappe pas à la personnalisation du silence qui est une façon de moduler l'énonciation, de sous-entendre sa portée par un retard, une mise en retrait, un temps de réserve.

L'une des pratiques grâce auxquelles, tant bien que mal, on s'accommode des affres quotidiennes; parler, non pour dire ce que l'on porte au fond de soi et que, pour peu que l'on ne soit pas seul, il y aurait lieu de confier puisque c'est cela qui oppresse dès le réveil, mais pour se libérer provisoirement $d u$ terrible faix en s'embarquant dans un commerce de paroles d'une toute autre veine. Comme s'il fallait le va-et-vient moi et toi du dialogue pour emporter les mauvais gravats... ${ }^{3}$

Il est possible d'étudier ces manifestations du silence: c'est l'objet d'une linguistique qui s'intéresse à la modélisation énonciative de l'acte de parole dans sa relation à la posture corporelle du sujet. Il n'importe pas à cette occasion de percevoir le silence à la faveur d'une interruption manifeste de la parole. L'enjeu est plus vaste puisque la proxémique du sujet de la conversation doit être étudiée afin de percevoir à quel moment le sujet donne une signification posturale au silence alors même qu'il parle. Une linguistique du silence, dans la mesure où elle s'intéresse aux formes énonciatives de l'oralité, peut correspondre à un tel projet. Ce n'est pas le cas, on s'en doute, du texte littéraire. Je prendrai comme source de cette réflexion le travail autobiographique de Michel Leiris afin d'indiquer de quelle manière la voix, sous la forme du chant, ou le cri sont les points de chute d'un silence soumis au refoulement puisque l'énonciation du silence a comme condition première de se manifester par la parole.

Il peut paraître paradoxal d'énoncer que la voix est l'opérateur sémiotique du silence. À suivre les écrits de Vladimir Jankélévitch sur la musique, ou les travaux de Paul Zumthor sur la question de l'oralité et de la voix ${ }^{4}$, il me semble que la sémiotisation de la voix loge le silence sous l'aspect d'un secret indicible. Sur ces questions, l'autobiographie adopte une perspective tout à fait singulière. Elle n'appartient pas à l'espace narratif dit traditionnel de la culture orale. Pourtant l'autobiographie, quelle qu'en soit la présentation singulière par tel ou tel auteur, exige de mettre un terme au silence afin d'énoncer la vérité du sujet. L'autobiographie est cette mécanique discursive bien huilée qui n'accepte pas de rompre avec la mainmise d'un discours autoréférentiel. L'obsession autobiographique concerne la déclamation des figures de soi. La figurativité est évoquée de manière persistante puisque le sujet revendique la pertinence de $s a$ diction au regard d'une instance que la psychanalyse nomme l'Idéal du Moi. Le silence ne serait-il pas dès lors le refoulé originaire de l'acte autobiographique, ce qui ne se dit pas et qui demeure à l'écart de l'énonciation?

L'œuvre autobiographique tardive de Michel Leiris me semble révélatrice de cette désémiotisation qu'implique le recours à la voix dans le cadre d'une réflexion sur le récit de soi ${ }^{5}$. Cette désémiotisation correspond chez Leiris au discours vocal qui se donne à lire sous l'aspect de l'informe sonore, de la brutalité pulsionnelle: force violente qui contribue à réduire le sens à néant.

Sentiment d'un trou vertigineux creusé soudain dans

l'écoulement des minutes quotidiennes, l'effroyable gêne causée par Antonin Artaud, apparemment point encore ravagé par le 
mal qui un jour motiverait son internement, mais donnant un échantillon de cri théâtral - hurlement émis à pleins poumons et d'une certaine durée - au cours d'une conférence prononcée à la Sorbonne pour un groupe d'études dont les problèmes de l'art moderne étaient le principal intérêt. 6

Leiris ne cesse dans ces écrits tardifs de revendiquer la puissance de la voix; c'est l'exemple de la forme mélodique qui inscrit la bipolarité du proche et du lointain incarnée par la figure maternelle:

La voix qui déployait ainsi quelque chose d'analogue à ce que

l'on nomme en cartomancie le grand jeu, je me rappelle

combien elle était ample, pure, puissante et veloutée. Mais le

souvenir que j'en ai, s'il me permet d'évaluer le trésor que

possédait ma tante Claire, n'y répond que d'une manière

formelle et n'a pas l'acuité voulue pour que je puisse,

mentalement, l'entendre à nouveau chanter. Peut-être cette voix

me touchait-elle de si près que je m'en laissais imprégner sans

presque l'écouter? Peut-être n'était-elle pour moi que l'une des

façons, entre autres, qu'avait de se manifester la créature

rayonnante que je ne parviens pas aujourd'hui à séparer $d u$

décor de ses épiphanies [...]. 7

La violence insupportable du cri qui énonce

l'éviscération de soi:

Expressif certes, mais en deçà de tout langage et trafiqué par nulle modulation, le cri à l'état pur, autrement dit le cri inarticulé (comme celui qu'arrache la torture, la terreur, la joie

folle, ou une grande surprise). Le cri: ensauvagement de la voix qui, retournée semble-t-il aux origines, perd son identité et, rendue à sa base biologique, ne peut plus être certifiée mâle ou femelle et se reconnaît à peine comme émanant d'un être humain. 8

Ce cri qui rejoint le silence dans sa proximité d'outre-tombe, Leiris l'aura rêvé en compagnie d'amis prestigieux. Bacon ne cesse de peindre l'éviscération du corps qui est la bouche emmurée du silence. Artaud profère de sa voix de fausset Pour en finir avec le jugement de Dieu, cri intolérable puisque Radio France préféra interdire l'émission et laisser au poète le domaine du silence. Cette désémiotisation traduit un processus radical puisqu'elle interroge les frontières de la voix et du silence. Lorsque Leiris fait référence à la voix, à sa continuité syntagmatique, ou encore à sa violence destructrice, il indique ce qui demeure insupportable dans la voix: la forme refoulée du silence. C'est le silence qui ne peut être toléré et qui condamne à parler - ou à écrire - inlassablement.

L'étude de l'acte autobiographique doit aborder cette question du silence qui tient lieu de refoulement originaire $^{9}$ si elle ne veut pas faire l'impasse sur des arguments qui font référence à la vérité ou à la fausseté de l'énoncé. Cette modalité de refoulement, qui est littéralement infigurable pour la psyché, permettrait d'ordonner, à la suite d'une partition pulsionnelle, les représentations mnésiques subséquentes qui forment l'appareil psychique. Il faudrait alors envisager la constitution de l'appareil psychique sous l'aspect d'une catastrophe première dont le «sens» demeurerait refoulé, ce qui justifierait la mise en jeu d'une activité sémiotique ${ }^{10}$. Le refoulement originaire serait ce «silence» que la psyché s'impose afin de pouvoir penser.

Cette interrogation, qui fait référence au processus de désémiotisation, est pertinente si l'on choisit d'examiner la portée du silence. Ce dernier n'est pas la contrepartie de la voix, pas plus qu'il n'est cette absence de signification qui s'oppose à la présence de la parole. Le silence n'est pas l'aveu d'un manque-àdire, l'impuissance du sujet à trouver matière pour ce qu'il souhaiterait voir prendre forme lors du discours. Cette désémiotisation prend naissance en un temps psychique où le sujet se voit rappelé à un moment antérieur de sa structure libidinale. La désémiotisation qui acquiert le statut de modalité signifiante fait appel au silence. La prostration, le mutisme, la sidération sont de diverses manières des opérateurs du silence qui se traduisent par le non-engagement dans l'acte de parole. Toute une psychopathologie, nourrie par l'histoire de la psychiatrie, s'est efforcée de rendre compte de cette attitude où le refus d'engager la parole est le témoignage d'une mise en retrait du sujet. Ainsi l'étude de l'autisme infantile ${ }^{11}$ met l'accent sur une aporie constitutive du sujet dans la mesure où la parole, qui acquiert le statut de maîtresignifiant, semble absente, forclose. Pourtant la 
psychanalyse ne cesse de souligner que ces silences chez l'enfant sont lourds de sens, qu'ils sont plus probants, dans leur subjectivité inconsciente, que l'acte de discours explicite.

Ce processus de désémiotisation est révélateur d'une "attaque contre le lien de pensée» qui rappelle les travaux du psychanalyste W.R. Bion, puisque le silence, associé aux éléments bêta, rompt la forme de la continuité langagière ${ }^{12}$. Cette rupture brutale prend l'aspect d'un impact catastrophique qui détruit à sa source la possibilité même de créer un contenant de pensée. Il existe par ailleurs une distinction de taille entre la classification nosographique des modes réfractaires d'accès au langage, dont le silence dit pathologique est la forme, et ce que la psychanalyse et la littérature peuvent percevoir du silence. Si certains psychanalystes entrevoient le silence comme un affect, l'accompagnement d'une représentation refoulée qui trouve difficilement accès à la parole, d'autres psychanalystes attribuent au silence une portée langagière et sémiotique de première importance ${ }^{13}$. Le silence est l'indication privilégiée de l'entrée réussie dans la relation analytique lorsqu'il prend la forme somato-psychique d'un apaisement, d'une capacité à se laisser dire par le langage inconscient plutôt que d'être un énonciateur forcené qui bute sur les interdits, les secrets de famille, les injonctions surmoïques.

Cette qualité de silence rejoint ce processus de désémiotisation qui se joue lors de la cure de manière plus ample à partir de l'empreinte transférentielle que crée l'analyste et de la régression favorisée par le "cadre» thérapeutique. Certaines cures sont des architectures offertes au silence. Après un temps d'épuisement et de sidération justifié par une parole qui s'acharne à tout dire, à déclamer la nécessité de l'entrée en analyse, le sujet-analysant déchoit et déchante du pouvoir interlocutoire qu'il s'était attribué. Le sujet se met alors à parler grâce au silence qui lui offre la possibilité de se créer un contenant psychique, ou encore, pour reprendre l'expression consacrée de Julia Kristeva, une chora qui a valeur d'inscription pulsionnelle archaïque. Le sujet sait alors, c'est là une trame puissante du processus analytique dans sa dimension transférentielle, qu'il n'a pas à parler ou à se taire. Cette logique bivalente est abandonnée au profit d'un acte de parole qui valorise la rencontre du silence et de la voix, la possibilité de faire entendre le silence dans la parole, de faire entendre la signification dans un silence maintenu.

La psychanalyse est très proche de la sémiotique puisqu'elle interroge la vie émotionnelle du langage dans sa dimension formelle, alors que la psychiatrie, du moins sous sa forme canonique, propose une axiologie des contenus psychiques sous l'aspect d'une symptomatologie. Le champ d'exercice de la psychanalyse nous permet de mieux comprendre la mise en jeu du silence. La psychanalyse est une science de la parole: on ne cesse de le répéter à la suite de l'énoncé révolutionnaire de Berthe Pappenheim qui faisait valoir à Joseph Breuer la portée de la «talking cure». Mais la psychanalyse n'est pas concernée par les seuls phénomènes d'interlocution qui se manifestent lors de l'échange transactionnel d'actes de parole. Affirmer que le langage est composé d'éléments discrets dont la mise en relation fait système perpétuerait une conception atomistique et réductrice de l'inconscient. Les travaux de Bion sur la destruction du lien de pensée, les recherches de Piera Aulagnier sur la constitution du processus originairepictographique, les travaux de Lacan sur l'opération de forclusion dans la psychose ont pour enjeu d'expliquer que la convention discursive peut à tout moment faire l'objet d'une implosion massive dont le signe fragile est le silence. À cet égard, la psychanalyse adopte une perspective bifocale et réversible qui désigne bien la distance adoptée envers la psychiatrie. Cette dernière veut faire "sens» de manière à ce que la classification dynamique de la maladie mentale soit répertoriée. Il n'en va pas différemment dans le champ sémiotique car les enjeux axiologiques se sont eux aussi déplacés: l'intérêt pour une formalisation du sens, calquée sur le modèle des langues naturelles, la fiction idéologique de leur bon fonctionnement, laisse place à l'étude des manifestations discursives du «malentendu ", à l'analyse de l'hybridité des actes énonciatifs, à l'interrogation des coïncidences de 
l'affect ou de l'acte perceptif avec l'énonciation du sujet. Le silence est un enjeu contemporain de la réflexion sémiotique parce que la négativité de la parole entretient des liens formels avec le champ de la culture.

Mon objectif n'est pas de proposer une axiomatisation sémiotique du silence dans l'œuvre tardive de Michel Leiris, ce qui nous poserait des problèmes méthodologiques dont j'ai eu l'occasion de préciser l'ampleur. Dans cette œuvre, le silence est un opérateur discursif refoulé qui nourrit, sans doute de manière paradoxale, l'émergence de l'autobiographie.

Mon cogito professionnel: je parle donc j'existe - ce qui implique que je suis comme mort si nul ne veut plus m'entendre.

Etre entendu de quelqu'un, n'est-ce pas - hormis, menue monnaie, ce que requièrent les circonstances de tous les jours ma grande raison de parler puisque la violence, que comme beaucoup je voudrais réduire au mutisme, reste sourde à tous appels et n'est même pas embarrassée par les cris qui jaillissent à la gorge de ses victimes? ${ }^{14}$

Il est incongru de décréter que le silence est la source de l'autobiographie, que la topicalisation du silence amène le sujet à se dire, à se faire l'instrument, sinon le serf du langage. Pourtant, l'œuvre de Leiris ne cesse de ménager des lieux de silence qui sont aussi, j'aurai l'occasion d'y revenir, des réserves de secret. Il me semble que l'articulation du secret et du silence est propice à l'émergence de l'autobiographie ${ }^{15}$. Pour que celle-ci naisse, il faut que le secret soit une condition de la pensée. Il faut en somme que "quelque chose», inconnu, lié au refoulement originaire, soit interdit de discours pour mieux faire l'objet d'une énonciation. Leiris n'aura cessé de ménager dans son œuvre des lieux de silence. La passion poétique est d'abord éprouvée comme une rencontre impossible avec le réel. Il s'agit d'une rencontre sacrale qui mène à l'anéantissement.

Sans doute, l'écriture poétique est-elle, par rapport à l'écriture ordinaire, un peu ce qu'était dans l'opéra traditionnel l'aria opposée au récitatif (d'une part le chant ailé, d'autre part celui qui ne s'élève pas au-dessus du documentaire). Or, ce vers quoi spontanément j'ai tendu en interprétant des mots comme s'ils avaient été des termes de dictionnaire pris pour bases de développements rien qu'esquissés et à peine ou pas même syntaxés quand je ne me bornais pas à associer à un autre le mot prélevé, celui-ci, comme n'importe quel vocable hormis ceux qui ne sont que rouages $d u$ discours, possédant par sa structure des antennes qui le relient à au moins un vocable de la lignée autre, de sorte qu'en suivant cette pente on pourrait, obsédé, acharné à parcourir le vocabulaire entier ou - si je serre l'impression de plus près - s'aventurer jusqu'à se perdre dans un jeu de miroirs qui se renvoient les uns aux autres en d'innombrables réverbérations, découvrir le sens dernier, n'est-ce pas vers quelque chose qui, loin de n'être que balbutiement agile, serait à l'écriture poétique ce qu'avec leurs escarpements les vocalises représentent par rapport aux parties plus unies de l'aria ? 16

D'où le rôle salvateur de la prose autobiographique qui a dans l'œuvre de Leiris une efficacité réparatrice puisqu'elle préserve le sujet d'un danger de mort.

Ce silence maintenu sur la poésie, qui sera levé au moment de la rédaction des ouvrages tardifs, je pense notamment au Ruban au cou d'Olympia et à Langage tangage ou ce que les mots me disent, correspond d'ailleurs à une réflexion renouvelée sur l'opéra. Cette passion poétique fait de plus jouer, sous une forme tragique qui est associée à l'opéra, les lieux réservés de la conjugalité. Leiris, on le sait peut-être, aura été d'une discrétion absolue, du moins pour la narration autobiographique, à propos de son mariage avec Zette (Louise Godon) et des secrets de famille qui y étaient associés ${ }^{17}$. Ce souci de discrétion peut sembler anodin. Mais ce jugement rapide masquerait l'importance du secret qui traduit chez Michel Leiris la nécessité impérieuse de garder le silence sur l'archè à la source du projet autobiographique. L'acte d'écrire sur l'opéra permit de rejouer la rencontre avec la féminité qui fut déterminante pour Leiris puisqu'elle faisait voir une nudité à la fois conviée et crainte ${ }^{18}$. Dans cette passion pour l'opéra, n'y avait-il pas le désir de masquer le silence de la mort, d'imposer une destinée qui avait encore une fois l'aspect d'une mécanique bien huilée? N'y avait-il pas dans cette nécessité de donner voix au récit autobiographique le souci de payer son dû à une féminité entravée par 
l'obsession de la castration, du manque-à-dire, du phallicisme narcissisé qui traverse aussi cette œuvre?

La voix ne devenait-elle pas alors la figure magnifiée d'un signifiant non châtré, la naissance possible par le chant et son incarnation dans une œuvre hantée par la dimension posthume de l'écrit? Cette naissance par la voix ne prenait-elle pas d'ailleurs la forme sublimée d'une procréation dont la charge corporelle et sexuelle était atténuée par la traduction de la voix et du silence en projets d'écriture? N'y avait-il pas à la faveur de la solitude contemplative de l'écrivain l'exercice d'un déni du féminin mis à jour sous l'aspect singulier d'une voix: objet pulsionnel dont la forme sonore est enivrante et qui présente une tragédienne seule sur scène, aimée pour sa voix, ce signifiant énigmatique, immatériel qui est un énoncé de jouissance?

Cristaux pointant à des hauteurs glaciales dans la noirceur de

l' "Air de la Reine de la Nuit», pépiements d'oiseau éperdu quand l'air dit «de la folie» de Lucia di Lammermoor vient à prendre son envol. [...] Indicible? Ineffable? Je ne gonflerai mes joues d'aucun de ces grands mots pour exprimer ce à quoi je crois atteindre en ces moments où, subjugué par l'apparente frivolité de la prouesse vocale, je ne vis guère que par et pour l'oreille. Simplement, m'abstenant d'user d'un terme négatif qui ne suggère l'infini que par sa vacuité même et que notre bouche a sottement plaisir à boursoufler, j'affirmerai qu'en l'espèce la chose à dire est expressément dite, mais l'est par la musique avec les sons pénétrants - qu'aucune entente seconde n'émousse - de son langage à elle qui, sans qu'on puisse sauf mysticisme prétendre qu'elle creuse le ciel comme l'a écrit (splendidement) un merveilleux poète et esthéticien de l'époque romantique, pallie avec assez d'éclat pour nous combler la carence du langage que tout petits nous avons appris et que plus âgés nous parlons et écrivons, incapable quant à lui de nous conduire jusqu'à nos derniers recoins. 19

Le silence de l'écrivain, fervent autobiographe, ne rencontrait-il pas alors la solitude du chant dont Leiris énonce la nudité puisqu'il permet d'entrevoir ce qui ne se dit pas? L'autobiographe, en témoigne Leiris, laisserait chanter son écrit afin d'échapper à la mort. Le bavardage autobiographique ne serait qu'un leurre commode permettant de masquer l'omnipuissance de la mort. Michel Leiris vieillissant aura été emporté par la vitalité d'une écriture qui ne se satisfaisait plus du rituel tauromachique, ou de cette autre passion que fut la peinture. Dans l'œuvre de Leiris, le monde visible s'effiloche peu à peu et la voix, garant de la précarité de la tradition orale, devient la preuve même de la validité du discours autobiographique. Cette voix-témoin, Leiris ne cesse de la retrouver, à la fin de sa vie, sous la forme de la sœur aînée Juliette qui ne peut plus donner source au récit, l'authentifier. Cette voix, qui devient de plus en plus faible alors que Leiris vieillit, habite l'œuvre de façon souterraine. Il ne peut plus demander à sa sœur de rompre le silence amnésique de la mémoire autobiographique, de lui indiquer que cet événement précis s'est bien joué alors.

18 octobre

"Hallucination» dont ma sœur est le jouet et dont elle m'a parlé ce matin au téléphone (non en s'inquiétant, mais en trouvant cela «intéressant»). Ainsi, au cours d'une promenade en forêt (?), elle aurait vu un château Louis XIII qui n'existe pas. De même, assistant avec des amis à un concert (?), elle aurait vu le chef d'orchestre à demi caché par un buisson et, derrière lui, toute [une] ville se déployer. Je ne mets pas en doute la véracité de ces histoires qu'elle me raconte mais [lui] dit simplement que, étant donné sa mauvaise vue, elle doit «interpréter» ce que ses yeux lui fournissent. Réflexion faite, je me demande si son grand âge - quatre-vingt-quinze ans - ne la porte pas à mêler rêve, souvenir et réalité? 20

Michel Poizat souligne dans L'Opéra ou le cri de l'ange ${ }^{21}$ que la théorisation du silence est soumise à de multiples interprétations. On peut faire valoir la préséance du silence sur la voix, comme si le sujet était d'abord habité par un silence qui possédait ensuite une fonction signifiante. Le silence serait pour cette raison un signifiant énigmatique qui rappelle ce que le psychanalyste Jean Laplanche a pu énoncer de la valeur du signifiant parental tel qu'il est modélisé par le désir de l'enfant ${ }^{22}$. Ce signifiant énigmatique serait découpé par la voix, ce qui lui permettrait de faire jouer une première mise en séquence du langage. 
Vladimir Jankélévitch propose de son côté l'interrelation du silence et de la voix. À la faveur d'un jeu favorisant l'alternance du silence et de la voix, le langage émerge sous l'aspect d'un signifiant transitionnel. Celui qui se meut dans le monde intemporel du silence est déjà porté par une voix intérieure qui donne matière à penser. Le silence n'est donc pas un moment originaire qui offre son fondement à la parole. Il n'est pas cet univers informe qui permet au signifiant d'assurer un travail de découpe et de mise en relief.

À vouloir opposer de manière réductrice ces polarités, le théoricien est condamné à privilégier une perception schématique du langage. Le silence appartiendrait au monde de la nature; il serait l'incarnation vive d'un monde non altéré par une présence langagière qui solliciterait par la suite un énonciateur. Quant à la voix, elle serait la présence pure du langage, sa manifestation irréductible. En somme, la «voix» figurerait l'entrée dans la culture à partir de laquelle le sujet déclame son appartenance au collectif. On perçoit que le propos tenu à cette occasion est restrictif. Le silence appartiendrait au royaume des forces. Il serait la manifestation d'un signifiant dont l'antériorité manifeste signerait son appartenance au monde pulsionnel. Le silence, tout comme la mort si l'on suit la réflexion de Freud à propos de la non-représentation de notre finitude, serait un acte sémiotique à la fois inaugural et terminal. Le sujet naîtrait du silence auquel il contribuerait par ailleurs à donner voix, de la même manière que le sujet retourne au silence, royaume des forces et règne de l'inanimé. Constatons cette difficulté à articuler une réflexion sur le silence lorsqu'elle actualise les présupposés habituels: le silence serait l'envers ou l'avers de la voix, il en figurerait le doublet nécessaire. Cette mise en jeu bipolaire n'est certes pas fausse. Elle a seulement le démérite d'être générale, de réduire l'articulation de la voix au silence sous un mode qui ressemble à une construction schématique.

Une perspective sémiotique qui traite de cette question du silence adopte un autre point de vue. Elle interroge les «transformations» du texte littéraire à la faveur de l'inscription privilégiée d'un silence dont il faut retenir par ailleurs qu'il est toujours textualisé sous la forme d'un effet de silence dont on ne peut négliger la portée signifiante. En somme, l'effet de silence, qui traduit bien un processus de désémiotisation archaïque, est perçu grâce à une «voix» dont l'énonciation est moulée par l'empreinte du récit. La perspective est différente dans des domaines artistiques où la convocation de la voix s'offre comme un "acte» à entendre, par le biais de l'instrumentation technologique. C'est bien sûr le cas de la musique, des relais médiatiques traditionnels que sont la radio et la télévision, des archives sonores... Le silence y apparaît dans sa densité signifiante puisque la voix marque le silence de son empreinte.

Le texte littéraire ne peut pas restituer cet acte, pas plus qu'il n'en est la répétition différée. Le texte littéraire ne sait pas dire le silence, si ce n'est par une modulation métaphorique qui donnera à entendre ce signifiant de démarcation qu'est l'effet de silence. Chez Leiris, la voix permet de faire entendre cet acte sémiotique du silence. La puissance affective dont témoigne sa passion pour l'opéra est une façon de créer une oralité différée où le silence trouve sa place. Chez lui, le projet de donner voix au récit par le biais de l'opéra n'est pas éloigné d'une volonté de transfiguration par l'écriture. Il ne cessera de s'engager sur ces voies de traverse qui renouent avec la poésie.

Donner une voix au récit, ce serait alors témoigner de la distance entre l'écriture du jeune Leiris dans L'Âge d'homme et le discours sans voix que l'on retrouve dans les textes de la Fin: en témoignent À cor et à cri, ou bien Opérratiques. L'Âge d'homme se veut une scarification déclamatoire qui conteste le vérisme de l'autobiographie traditionnelle. À cor et à cri est la fragile saisie de la voix d'un autobiographe qui a perdu confiance dans le monde visible. Dans ce dernier livre, l'obsession du récit de soi bute sur le versant mélodique de la voix. "Quelque chose» qui n'est pas de l'ordre du signifiant linguistique et de sa formalisation symbolique trouve à se faire entendre. 
«Quelque chose» qui n'est pas l'affect, s'il fallait y percevoir la profération somato-psychique d'un indicible qui échappe au langage. Revenons à cette figure du signifiant transitionnel. La voix n'appartient pas à la convention du langage articulé lorsqu'elle est profération, cri ou vocifération glossolale. Quant à l'affect de la voix, il signifie de la même manière que le silence par l'envahissement interne qu'il crée puisqu'il donne forme à l'informulé. L'autobiographie, en témoigne l'œuvre de Leiris, ne serait-elle pas le lieu de rencontre de ce signifiant transitionnel: voix du silence et silence de la voix?

\section{N O TES}

1. M. Leiris, À cor et à cri, Paris, Gallimard, 1988, p. 112-113.

2. Je suis librement la pensée du psychanalyste W.R. Bion sur cette question. Bion, qui n'a cessé de proposer une théorie exigeante du processus sémiosique à propos de l'acte de pensée, écrivait: « La perspective réversible est un signe de douleur; le patient renverse la perspective pour rendre statique une situation dynamique. Le travail de l'analyse consiste à restituer son caractère dynamique à une situation statique et permettre ainsi à cette situation de se développer. Comme je l'ai indiqué dans le chapitre précédent, le patient manœuvre de manière à tomber d'accord avec les interprétations de l'analyste. Comme les interprétations de l'analyste ne permettent pas cela et comme le patient ne dispose pas toujours d'une agilité d'esprit suffisante pour faire coïncider l'interprétation avec un renversement subit de perspective, il emploie un outillage renforcé par le délire et l'hallucination ». Éléments de la psychanalyse, Paris, P.U.F., 1979 (1963), p. 60-61. Bion ne fait pas référence à cette occasion à la définition conventionnelle du signe linguistique. Il pose cependant, à la faveur de ce qu'il nommera des "transformations", les conditions sémiosiques qui permettent de penser le changement, ou encore de sombrer dans l'hallucination.

3. M. Leiris, À cor et à cri, p. 51.

4. P. Zumthor, Introduction à la poésie orale, Paris, Seuil, 1983; V. Jankélévitch, La Musique et l'Ineffable, Paris, Seuil, 1983.

5. Cette désémiotisation implique la contestation, au sein même du discours, de la forme qui le constitue. La mise en jeu du silence, comme forme désémiotique de la voix, me semble correspondre à un tel projet dans l'œuvre terminale de Michel Leiris.

6. M. Leiris, À cor et à cri, p. 23.

7. Idem, La Règle du jeu III. Fibrilles, Paris, Gallimard, 1966, p. 141.

8. Idem, À cor et à cri, p. 23

9. On s'en tiendra pour l'exposé à la définition qu'en donne un dictionnaire connu: «Processus hypothétique décrit par Freud comme premier temps de l'opération du refoulement. Il a pour effet la formation d'un certain nombre de représentations inconscientes ou "refoulé originaire". Les noyaux inconscients ainsi constitués collaborent ensuite au refoulement proprement dit par l'attraction qu'ils exercent sur les contenus à refouler, conjointement à la répulsion provenant des instances supérieures ". J. Laplanche et J.-B. Pontalis, Vocabulaire de la psychanalyse, Paris, P.U.F., 1967, p. 396-197.

10. On lira à ce sujet J.L. Goyena: «Nouvelles idées, nouvelles théories et changement catastrophique», dans Association française de psychiatrie (Collectif), W. R. Bion, une théorie pour l'avenir, Paris, Métailié, 1991, p.93-105.

11. On consultera sur cette question les travaux essentiels de D. Meltzer et F. Tustin.

12. «Dans l'hallucinose, les transformations sont en rapport avec un "désastre" ou une "catastrophe" primitifs, dans lesquels les contenus émotionnels des choses-en-soi (éléments [bêta]) n'ont pas trouvé un contenant (mère capable de rêverie) qui puisse les contenir, (les accueillir) et les transformer. La "terreur sans nom" (ou "panique psychotique”, comme Bion l'appelle aussi), renvoyée au nourrisson dans ces conditions, constitue un mode de fonctionnement mental dans un domaine dont les dimensions sont infinies et qui ne peut pas servir de contenant. Face à un tel état, la personnalité psychotique adopte des défenses destinées à éviter la panique, en éliminant les fonctions capables de l'enregistrer ou même de la percevoir ".

L. Grinberg, Introduction aux idées psychanalytiques de Bion, Paris, Dunod, coll. «Psychismes", 1976, p. 84.

13. Le livre d'A. Green, Un Discours vivant (Paris, P.U.F., coll. «Le fil rouge", 1973), demeure encore aujourd'hui sur cette question un ouvrage de référence.

14. M. Leiris, À cor et à cri, p. 73 .

15. On lira à ce sujet, bien que le propos ne fasse pas référence de façon explicite à l'autobiographie, le beau texte de J.L. Goyena: «L'intime et le secret. À propos de la transmission de la psychanalyse et du rituel des sociétés secrètes ", dans Psychanalystes (La psychanalyse et son establishment: les paradoxes de la transmission), Paris, hiver 1993-94, $n^{\circ} 48$, p. 105-119. Quant à Leiris, son métier d'ethnologue l'amena à rédiger La Langue secrète des Dogons de Sanda (Paris, Institut d'ethnologie, 1948 [rééd. Paris, J.-M. Place, 1992]). Faut-il s'étonner de cette mise au secret du nom de Louise Godon, femme de Leiris, à la faveur d'un projet ethnographique qui relance la quête autobiographique? 16. M. Leiris, Langage tangage ou ce que les mots me disent, Paris, Gallimard, 1985, p. 112-113.

17. «Lors de l'enquête généalogique qui fut menée après sa mort et qui, motivée par l'édition même de ce journal dont il m'avait chargé, me conduisit à consulter des pièces de l'état civil, il est apparu que Zette, la femme de Michel Leiris, n'était pas la sœur de l'épouse de Kahnweiler, mais la fille naturelle de celle-ci, c'est-à-dire la belle-fille de Kahnweiler - non point sa belle-sœur comme cela fut maintes fois dit et écrit. $\mathrm{Si}$ bien que les personnes qui, en certains endroits de La Règle du jeu ou dans les livres postérieurs, sont identifiées par les termes de beau-frère, de belle-sœur ou de søur de Zette sont en réalité les beau-père, bellemère de Leiris, ou mère et tante de sa femme - Leiris n'étant plus le beau-frère mais le gendre des uns ou le neveu par alliance des autres. On peut légitimement s'interroger sur la fonction d'un tel secret et sur la signification de ce qui apparaît bien, en dépit de la recherche de la vérité à laquelle Leiris s'est livré opiniâtrement, comme une «cachotterie» de sa part. J. Jamin, «Présentation» du Journal de M. Leiris (1922-1989), Paris, Gallimard, 1992, p. 16.

18. Selon C. Maubon: "C'est dans la gloire des rôles interprétés par Tante Lise - Carmen, Salomé, Électre, Dalida, Tosca - que Judith, faucheuse de têtes et de sexes, peut alors apparaître, tour à tour "déesse sanguinaire", "magnifique et tentante créature" (p.92), "fille implacable et châtreuse" (p.95), "bacchante échevelée" (p.96), "tueuse" (p.97), et "ensorceleuse lascive" (p.99) \%. C. Maubon commente L'Âge d'homme de M. Leiris, Paris, Gallimard, 1997, p. 79.

19. M. Leiris, Langage tangage ou ce que les mots me disent, p. 114.115. 20. M. Leiris, Journal (1922-1989), p. 761.

21. M. Poizat, L'Opéra ou le cri de l'Ange, Paris, A.M. Métailié, 1986. 22. J. Laplanche, Nouveaux Fondements pour la psychanalyse, Paris, P.U.F., 1967. 